\title{
Dictionary Based Reconstruction of the 3D Morphology of Ebola Virus
}

\author{
Alaa AlAfeef ${ }^{1,2}$, W. Paul Cockshott ${ }^{1}$, and Ian Maclaren ${ }^{2}$ \\ ${ }^{1}$ School of Computing Science, University of Glasgow, Glasgow, G12 8QQ, UK \\ ${ }^{2}$ SUPA School of Physics and Astronomy, University of Glasgow, Glasgow, G12 8QQ, UK
}

The filoviruses Ebolavirus (EBOV) and Marburgvirus cause fatal hemorrhagic fevers in humans and other mammals, known as Ebola virus disease (EVD), with mortality rates approaching 90\% [1] which makes it listed as world health organization risk group 4 pathogen. Ebola virus caused the 2013-2015 epidemic in West Africa, which has resulted in at least 9,268 confirmed deaths and 23,034 suspected cases as of 18 Feb 2015 [2]. Consequently, considerable effort is focused on developing therapeutics and vaccines to prevent infections.

Cryo electron tomography (cryo-ET) is now an increasingly important technique for obtaining structural details of complex viral component organization at subnanometer resolution. Specifically, with reference to EBOV, Cryo-ET can be used for studying the arrangement of the internal viral components of EBOV such as the glycoprotein spikes (GP) on the surface of EBOV which are the target of multiple neutralizing antibodies [3]. This is vital for improved understanding of the principles of infection, strategies to combat the viral infection.

Electron Tomography (ET) typically involves the acquisition of a set of two-dimensional projection images by rotating the specimen over a tilt range (typically restricted to $+/-70^{\circ}$ ) using bright field Transmission Electron Microscopy (BF-TEM), followed by alignment and reconstruction using established algorithms to reconstruct a 3D volume that represents the physical morphology of the specimen under investigation. However, reconstruction still suffers from distortion (especially the missing wedge artifacts) due to the limited range of angles sampled. Also, specifically for tomography on sensitive biological materials, low signal-to-noise ratio is a problem due to the need to limit the electron dose over the entire tilt series, resulting in noisy 3D representations.

Compressed sensing (CS) has recently been applied to ET, and such approaches are both more noise tolerant and reduce out of plane distortions from the missing wedge [3]. In this work, we reconstruct a cryo-tilt series acquired at approximately $2^{\circ}$ intervals from $\pm 60^{\circ}$ of an Ebola entry-competent virus-like particles (VLPs), using datasets published previously in reference [6], using a new compressed sensing based algorithm, DLET [5]. These results are compared to a 3D reconstruction of the same datasets obtained using the well-known SIRT algorithm. DLET utilizes a dictionary-based learning approach and is very noise-tolerant, allowing reliable reconstruction from far fewer projections than are normally required by traditional ET methods.

Figure 1 shows a comparison between SIRT and DLET for reconstruction using tilt series 1 . Please note that this and the subsequent figure are produced without any segmentation to show the raw results of the reconstruction and to emphasize the clear difference between the results of the two methods. In Figure 1 it is clear, and it can be noted that DLET gives a noticeable much clearer visibility of the details of the virus and its morphology, including the clearly visible GP spikes surrounding particles it. Figure 2 shows a volume rendering of another reconstruction for a second other tilt series. Here the DLET shows a more much clearer reconstruction with a significant decrease of reconstruction noise. Consequently, 
we show that the use of this dictionary-based reconstruction algorithm gives major benefits for the reconstruction the $3 \mathrm{D}$ volume of viruses from ET datasets of limited tilt range, relatively low signal to noise, and low contrast levels. It is anticipated that the application of this technique will present major advantages in discerning the nanoscale details of virus structure and infection behavior as compared to established tomographic reconstruction algorithms.

\section{References:}

[1] Feldmann, Heinz, and Thomas W. Geisbert,The Lancet 377.9768 (2011), p.849.

[2] World Health Organization, "Ebola Situation Report - 18 February 2015" (2015).

[3] Qiu, Xiangguo, et al., Sci. Transl. Med., 4.138 (2012), p.138ra81.

[4] Saghi, Zineb, et al., Nano letters 11(2011), p.4666.

[5] A. AlAfeef et al., J. Phys.: Conf. Ser 522 (2014), P.012021.

[6] Tran, Erin EH, et al., Journal of virology, 88.18 (2014),p.10958.

[7] AAA acknowledges funding for a PhD studentship from the Lord Kelvin/Adam Smith Scholarship of the University of Glasgow. This work was made possible by generous help from Erin E. H. Tran and Sriram Subramaniama from National Institutes of Health, Bethesda, Maryland, USA.
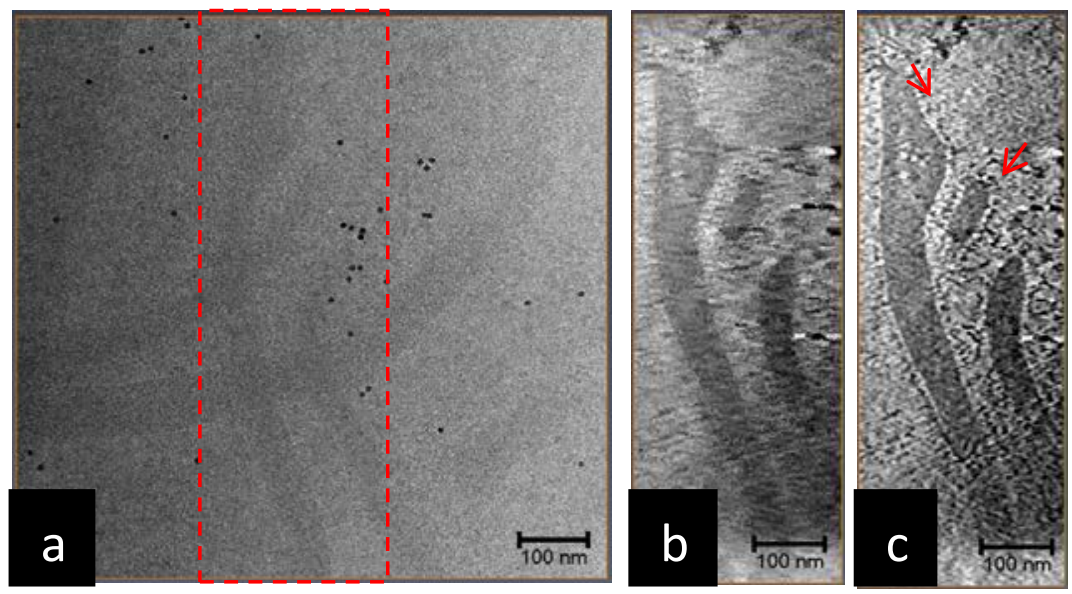

Figure 1. a) $0^{\circ}$ Projection of Ebola-VLP b)XY-Orthoslice obtained through the constructed volume using SIRT and c)DLET. The GP spikes are clearly visible surrounding particles as indicated by arrows
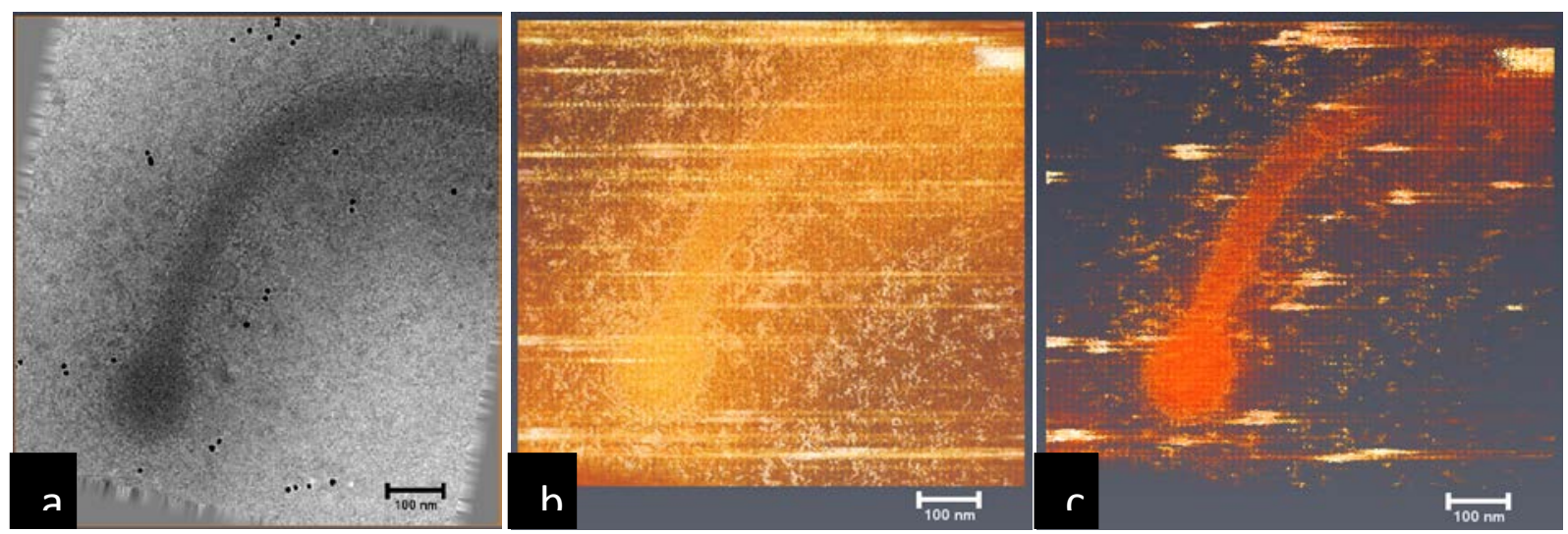

in (c).

Figure 2. a) $0^{\circ}$ Projection of Ebola-VLP and gold fiducial markers (b) Volume rendering of reconstructed volume using SIRT and (c) DLET. Smearing due to markers is reduced in (c). 